\title{
Second-trimester abortion: women often lack the choice they should be offered
}

\section{Mary Pillai, ${ }^{1,2}$}

${ }^{1}$ Gloucestershire Care Services NHS Trust, Gloucester, UK ${ }^{2}$ Department of Obstetrics, Gloucestershire Hospitals NHS Foundation Trust, Gloucester, UK

\section{Correspondence to} Dr Mary Pillai, Gloucestershire Hospitals NHS Foundation Trust and Gloucestershire Care Services NHS Trust, Gloucestershire Royal Hospital, Gloucester GL1 3NN, UK; mary. pillai@nhs.net

Received 4 September 2017 Revised 8 September 2017 Accepted 12 September 2017

\section{Linked}

- http://dx.doi.org/10.1136/ jfprhc-2016-101652

CrossMark

To cite: Pillai M. J Fam Plann Reprod Health Care 2017;43:249-251.
In this issue, Koh et al. ${ }^{1}$ from Singapore report on their randomised study of three vaginal prostaglandin regimens for mid-trimester termination of pregnancy. ${ }^{2}$ This article addresses gaps in knowledge both of the optimum dose of the most commonly used agent, misoprostol, and of the relative efficacy of the product licensed for this indication, gemeprost. But the question of whether medical termination is, in fact, the most suitable approach for termination after the first trimester remains open to debate.

Over the past 20 years, the overall abortion rate in England and Wales, where figures are believed to be compiled with a high degree of accuracy, has plateaued at around 16/1000 women aged 15-44 years. In 2016 , over $80 \%$ of procedures were undertaken at under 10 weeks' gestation, with a continuing rise in the proportion utilising early medical abortion. ${ }^{3}$ However, the proportion of abortions carried out at 13 weeks' gestation or above has remained static at around 8\% and is unlikely to change in the foreseeable future. Mid-trimester abortion will continue to be necessary for a range of reasons including some women's ambivalence about their decision, women not recognising their pregnancy due to contraceptive use or because they believe that they are infertile due to their age or medical factors, concealed pregnancies (particularly in teenagers), difficulty in engaging services due to mental health problems or learning difficulties, pregnancies that were initially wanted but where the woman's circumstances have changed, and where serious fetal abnormality has been diagnosed. ${ }^{4}$ The introduction of more efficacious screening programmes in the UK has resulted in a much higher proportion of fetal anomalies being diagnosed antenatally. However, for many of the anomalies detected by screening tests, including the range of anomalies detected by routine ultrasound scans, most of the pregnancies will have advanced to the second trimester by the time of diagnosis. Unfortunately the choices of method then available to those women requesting abortion are limited. ${ }^{5}$

Abortion in the UK is very safe, but the risks and complexity of the procedure increase with advancing gestation. Worldwide, 'late' procedures account for a disproportionate amount of abortion-related morbidity and mortality. Following liberalisation of abortion laws in the USA and much of Europe in the 1960 s and 1970s, the surgical procedure of dilatation and evacuation (D\&E) was developed. 'Medical' methods of second-trimester abortion were also developed, initially injecting hypertonic saline or urea into the amniotic sac, but these were soon replaced by much safer and more effective regimens using prostaglandins. The evidence base comparing surgical and medical second-trimester abortion is limited owing to difficulty in recruiting women to trials. The only randomised controlled trial (RCT) of surgical versus modern (non-instillation) medical abortion was stopped after 1 year, when $62 \%$ had declined participation, primarily due to a preference for D\&E. ${ }^{6}$ Although the data are limited, comparisons of procedures carried out at 13 to 24 weeks show significantly more complications with medical than with surgical termination $(>20 \% \mathrm{v} 4 \%) .{ }^{6-8}$ This reflects greater blood loss, more failed procedures and a high rate of surgical intervention for retained placental tissue.

Due to its speed, predictability and the expectation of significantly less pain, many women would choose surgery. This is reflected in UK national guidance. ${ }^{9}$ A 5 -year review of requests for termination for fetal anomaly in my own service, where a choice of medical or surgical termination is offered up to 17 weeks +6 days, showed that of 118 
consecutive eligible cases only two women requested a medical procedure. ${ }^{10}$ In one case this was influenced by the relative importance of autopsy and in the other the woman wished to avoid general anaesthesia.

There is, however, a large discordance between evidence and actual practice within the UK National Health Service (NHS) overall. ${ }^{11}$ It is rare for women to be offered a surgical procedure after the first trimester. This contrasts markedly with the independent sector in the UK, where surgical abortion has been the most common method used for mid-trimester procedures up to 24 weeks' gestation, and mirrors practice in the USA where D\&E is the predominant method of termination throughout the mid-trimester.

Abortion services in the UK now face a workforce crisis as a high proportion of the small number of surgeons who provide D\&E have reached, or are approaching, retirement. Gynaecological surgical training in the UK occurs almost exclusively within the NHS, but as few NHS gynaecological surgeons perform D\&E, there are almost no role models or opportunities for training. Those who do perform D\&E in the NHS frequently meet barriers from theatre staff who find the procedure psychologically distressing, owing to the need for extraction of recognisable fetal parts. Where this is combined with ultrasound guidance there is good evidence that the safety of the procedure is enhanced, but staff may find the imaging a further source of distress. If there is no ultrasound imaging, checking the fetal parts is required to clarify completeness of the procedure and this can be the most upsetting process of all.

So for most women in the UK diagnosed with a fetal anomaly, or requiring a mid-trimester abortion for any other reason, the lack of expertise to offer a choice of method within the NHS means that medical abortion will be the only option offered. ${ }^{5}$ As the independent sector becomes less able to source the required pool of skilled surgical providers, medical termination may increasingly become the default position for late terminations throughout the UK. Against that background, Koh et al's $\mathrm{RCT}^{2}$ provides useful information on different regimens for mid-trimester medical abortion. Unlike comparisons between surgical and medical procedures, comparisons of differing medical procedures provide a relative abundance of data, including a Cochrane Review comparing 36 RCTs. ${ }^{12}$ That review concluded that the optimal dose of vaginally administered misoprostol could not be determined as no RCTs could be identified. Koh et al's article addresses this gap but also includes a third comparison arm with gemeprost, which, unlike misoprostol, is licensed for this purpose and is still used in some countries. However, in the UK misoprostol, either alone or in combination with other agents, particularly mifepristone, has supplanted other methods because of its high efficacy, low cost and relative ease of use.

On a worldwide basis, the information in Koh et al's study is even more significant than for care within the UK, as mifepristone is not licensed in many countries. ${ }^{13}$ Medical abortion is not a complex procedure and is certainly safer than childbirth. So potentially a range of non-medical providers could deliver it. In resource-poor countries the relatively high cost of mifepristone and both the high cost of gemeprost and the requirement for its storage below $-10^{\circ} \mathrm{C}$ limit their use. So this additional evidence of the effectiveness of 4-hourly misoprostol alone (ie, without prior mifepristone) for second-trimester medical abortion is valuable. The study does, however, have two significant limitations. First, owing to local policy, the women were required to undergo a check surgical evacuation even if it appeared that abortion was complete. Surgery was therefore not avoided for the participants. Second, women with uterine scars (principally following Caesarean section) were excluded, so the study adds no data on safety and efficacy of abortion options for that group.

While there is strong evidence that for mid-trimester termination D\&E offers significant advantages over medical methods, in practice many practitioners will only be in a position to offer their patients a medical approach. For those services that can only offer medical abortion, and where mifepristone is not available, this study adds reassuring information about an effective medical regimen.

Competing interests None declared.

Provenance and peer review Commissioned; internally peer reviewed.

(C) Faculty of Sexual and Reproductive Healthcare of the Royal College of Obstetricians and Gynaecologists (unless otherwise stated in the text of the article) 2017. All rights reserved. No commercial use is permitted unless otherwise expressly granted.

\section{REFERENCES}

1 Koh DSC, Ang EPJ, Coyuco JC, et al. Comparing two regimens of in travaginal misoprostol with intravaginal gemeprost for second-trimester pregnancy termination: a randomised controlled trial. J Fam Plann Reprod Health Care 2017;43:249-51.

2 Koh DSC, Ang EPJ, Coyuco JC, et al. Comparing two regimens of in travaginal misoprostol with intravaginal gemeprost for second-trimester pregnancy termination: a randomised controlled trial. J Fam Plann Reprod Health Care 2017;43:249-51.

3 Department of Health. Abortion Statistics: England \& Wales 2016. 2017 https://www.gov.uk/government/uploads/system/ uploads/attachment_data/file/618533/Abortion_stats_2016_ commentary_with_tables.pdf (accessed 31 Aug 2017).

4 Ingham R, Lee E, Clements SJ, et al. Reasons for second trimester abortions in England and Wales. Reprod Health Matters 2008;16:18-29.

5 Fisher J, Lohr PA, Lafarge C, et al. Termination for fetal anomaly: are women in England given a choice of method? J Obstet Gynaecol 2015;35:168-72.

6 Grimes DA, Smith MS, Witham AD. Mifepristone and misoprostol versus dilation and evacuation for midtrimester abortion: a pilot randomised controlled trial. BJOG 2004;111:148-53. 
7 Kelly T, Suddes J, Howel D, et al. Comparing medical versus surgical termination of pregnancy at 13-20 weeks of gestation: a randomised controlled trial. BJOG 2010;117:1512-20.

8 Lohr PA, Hayes JL, Gemzell-Danielsson K. Surgical versus medical methods for second trimester induced abortion. Cochrane Database Syst Rev 2008;1:CD006714.

9 Royal College of Obstetricians and Gynaecologists (RCOG). The Care of Women Requesting Induced Abortion (Evidence-based Clinical Guideline Number 7). 2011 https://www.rcog.org.uk/globalassets/documents/ guidelines/abortion-guideline_web_1.pdf (accessed 31 Aug 2017).
10 Pillai M, Gazet C, Staddon J. Management of termination of pregnancy for fetal anomaly (BMFMS 18th Annual Conference Abstract). BJOG 2016;123S1:52.

11 Lyus R. Re: Comparing medical versus surgical termination of pregnancy at 13-20 weeks of gestation: a randomised controlled trial. BJOG 2011;118:1148-9.

12 Wildschut H, Both MI, Medema S, et al. Medical methods for mid-trimester termination of pregnancy. Cochrane Database Syst Rev 2011;1:CD005216.

13 Gynuity Health Projects. Resources: Map of Mifepristone Approvals. http://gynuity.org/resources/info/map-ofmifepristone-approvals/ (accessed 31 Aug 2017). 